\title{
C-BAND HIGH POWER RF GENERATION AND EXTRACTION USING A DIELECTRIC LOADED WAVEGUIDE*
}

\author{
F. Gao ${ }^{\#}$, M. Conde, W. Gai, R. Konecny, W. Liu, J. Power, Z. Yusof \\ ANL, Argonne, IL 60439, U.S.A \\ T. Wong, IIT, Chicago, IL, U.S.A.; C. Jing, Euclid TechLabs, LLC, Solon, Ohio, U.S.A.
}

\section{Abstract}

We report on design, fabrication, and beam test of a $7.8 \mathrm{GHz}$ power extractor recently conducted at the Argonne Wakefield Accelerator (AWA) facility. The wakefields are excited using an electron beam travels through a dielectric loaded waveguide, and the generated $\mathrm{RF}$ power is then subsequently extracted with a properly designed RF coupler. In the experiment, $30 \mathrm{MW}$ of output power is excited by a $66 \mathrm{nC}$ single electron bunch, and wakefield superposition by a train consisting of four bunches is also demonstrated. Both results agree very well with theoretical predictions. Future tests include more charge transmission for higher RF power, and more bunches in a train for longer RF pulses.

\section{INTRODUCTION}

Dielectric loaded accelerating structure has been studied for many years $[1,2,3]$, and collinear $[4,5,6]$ and also two beam based acceleration schemes [7] using the dielectric loaded structure have been experimentally demonstrated. Also a proof of principle experiment was performed using $21 \mathrm{GHz}$ dielectric tube at using the CLIC Test Facility [8]. However, in order to reach higher gradient using the two beam acceleration scheme, one has to increase the beam current and improve the transfer efficiency, and compactness.

The two beam acceleration (TBA) scheme studied here consists of two stages, a first stage which generates RF energy from a driving beam traversing in a decelerating structure, and then the RF energy is transferred to a second stage to accelerate an accelerated beam. The first stage, commonly known as a power extractor, is essentially a high power RF source. A power extractor based on a circular dielectric loaded waveguide has interesting properties:

- The frequency can be easily changed by choosing different dielectric constants, inner and outer diameters for the dielectric tube.

- The output power can be easily adjusted by changing the charge of the driving beam.

- The output RF pulse length can be adjusted by the number of bunches in a train.

In this paper, we report on an initial test of a $7.8 \mathrm{GHz}$ dielectric loaded power extractor. Given the planned future AWA operating beam parameters $(50 \mathrm{nC}, 60$ pulses), this device is capable of generating $280 \mathrm{MW}$ power that can be used as a general RF source to do high

\footnotetext{
*Work supported by the US Department of Energy under contract No. W-31-109-ENG-38.

\#fgao@hep.anl.gov
}

power/gradient tests on accelerating structures, such as breakdown tests on a dielectric loaded waveguide.

\section{STRUCTURE DESIGN}

The several main challenges in the design are how to generate wakefield power as high as possible, and how to extract the power efficiently with properly designed couplers. Also, the structure should be easy to fabricate and assembly.

\section{Deceleration Tube Design}

In the current power extraction design, the $\mathrm{TM}_{01}$ mode is chosen as the operation frequency for beam deceleration, and the tube parameters are listed in Table 1. Since the frequency of the linac at the AWA facility is $1.3 \mathrm{GHz}$, the synchronous frequency of the tube (at which the phase velocity of the $\mathrm{TM}_{01}$ mode is equal to the speed of light in free space $c$ ) is chosen to be $7.8 \mathrm{GHz}$, the $6^{\text {th }}$ harmonic of the linac frequency. The dielectric material is Corderite with a relative permittivity 4.6 , provided by Trans-Tech. The inner diameter is set according to the typical transverse size of the driving beam, and the outer diameter is adjusted to move the synchronous frequency to $7.8 \mathrm{GHz}$. The length is chosen to be long enough for effective interaction, also not too long to allow most particles to pass through. The theoretical drain time is $2.9 \mathrm{~ns}$, but in MAFIA simulation it is $2.2 \mathrm{~ns}$, this is due to the effect of the metal cutoff plug at the upstream end.

Table 1: Deceleration Tube Parameters

\begin{tabular}{|l|l|l|}
\hline$f$ & $7.8 \mathrm{GHz}$ & Frequency \\
\hline$I D$ & $12.04 \mathrm{~mm}$ & Inner diameter \\
\hline$O D$ & $22.34 \mathrm{~mm}$ & Outer diameter \\
\hline$L$ & $266 \mathrm{~mm}$ & Interaction length \\
\hline$\varepsilon_{r}$ & $4.6($ Corderite $)$ & Relative permittivity \\
\hline$\beta_{g}$ & 0.23 & Fractional group velocity \\
\hline$t_{d}$ & $2.2 \mathrm{~ns}$ & Drain time \\
\hline$Q$ & 2745 & Quality factor \\
\hline$r / Q$ & $6.09 \mathrm{~K} \Omega / \mathrm{m}$ & r over Q \\
\hline
\end{tabular}

The excited gradient and power at the downstream end of the tube are determined by both the tube parameters and the beam parameters. From MAFIA simulation, with $100 \mathrm{nC}$ single bunch excitation, the induced gradient will be $18 \mathrm{MV} / \mathrm{m}$, corresponding to $79 \mathrm{MW}$ of power generated. If a bunch train with a bunch frequency $1.3 \mathrm{GHz}$ and $50 \mathrm{nC}$ charge per bunch is used as the driving beam, the excited gradient will be $34.5 \mathrm{MV} / \mathrm{m}$, corresponding to $280 \mathrm{MW}$ of 
power generated. In the bunch train excitation, at least four bunches are needed for the gradient and power to reach the above values.

\section{$T M_{01}-T E_{10} R F$ Coupler Design}

In order to extract wakefield power to a rectangular waveguide, a $\mathrm{TM}_{01}-\mathrm{TE}_{10} \mathrm{RF}$ coupler is designed with CST Microwave Studio. Figure 1 shows the geometry of the coupler, in which the dielectric loaded tube surrounded by a vacuum region at the downstream end, then connected to an impedance matching section, and finally coupled to a WR112 rectangular waveguide. The beam hole at the downstream end is cutoff for the $7.8 \mathrm{GHz} \mathrm{TM}_{01}$ mode.

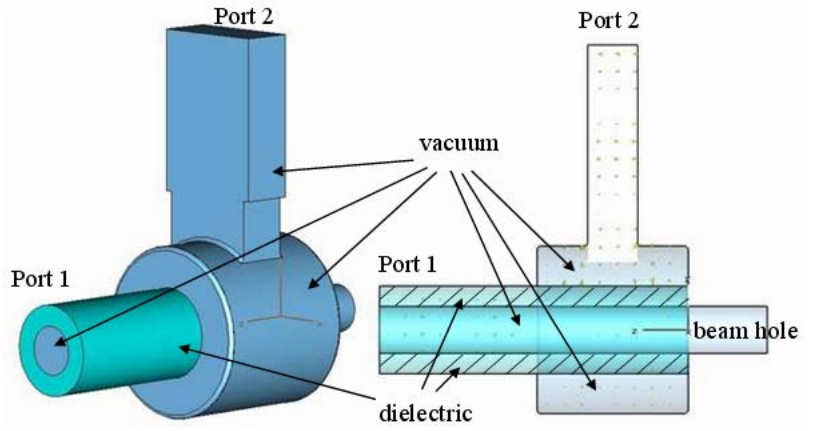

(a)

(b)

Figure 1: Geometry of the $\mathrm{TM}_{01}-\mathrm{TE}_{10}$ coupler (a) full view; (b) cutplane.

The coupler is tested with a HP8510C network analyzer, where a mode launcher is used to convert the TEM mode in the coaxial cable to the $\mathrm{TM}_{01}$ mode in the dielectric loaded tube. The simulated and measured insertion losses and reflections are plotted in Figure 2. The figure shows that at $7.8 \mathrm{GHz}$, the insertion loss $\mathrm{S}_{21}$ is $-0.41 \mathrm{~dB}$, which means the power coupling efficiency is $91 \%$.

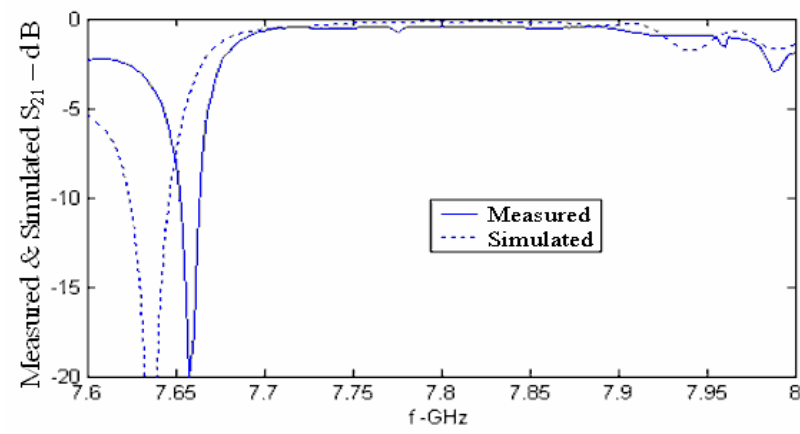

(a)

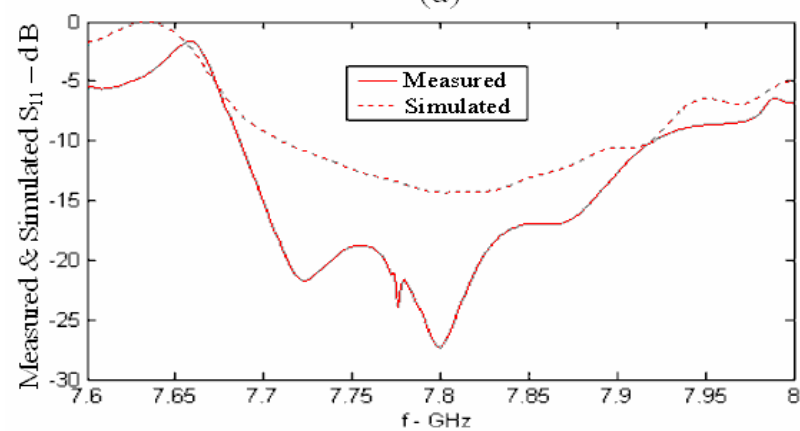

(b)

Figure 2: Measured and simulated $S_{21}$ and $S_{11}$ : (a) insertion loss $\mathrm{S}_{21}$; (b) reflection $\mathrm{S}_{11}$.

\section{BEAM EXPERIMENT}

The AWA beam line is able to deliver up to $160 \mathrm{nC}$ charge in a single Gaussian bunch out of the gun, with an rms bunch length $2 \mathrm{~mm}$. After a linac section, the beam energy reaches $14 \mathrm{MeV}$. This power extractor was installed in the beam line for experiment as shown in Figure 3. When a beam is sent through the power extractor, the excited RF power is coupled into a rectangular waveguide, where a bidirectional coupler with a coupling coefficient $-65 \mathrm{~dB}$ is used for signal detection. Both the forward and backward signals are sent to a Tektronix $15 \mathrm{GHz}$ digital real time oscilloscope for the measurement. After the bidirectional coupler, ideally a high vacuum high power load is needed to absorb the RF power. However, since such a load is not available at $7.8 \mathrm{GHz}$, alternatively a shorted waveguide is used to provide a time delay of $14 \mathrm{~ns}$ for signal identification. The vacuum inside the power extractor reached $7 \times 10^{-9}$ Torr after ten days of pumping.

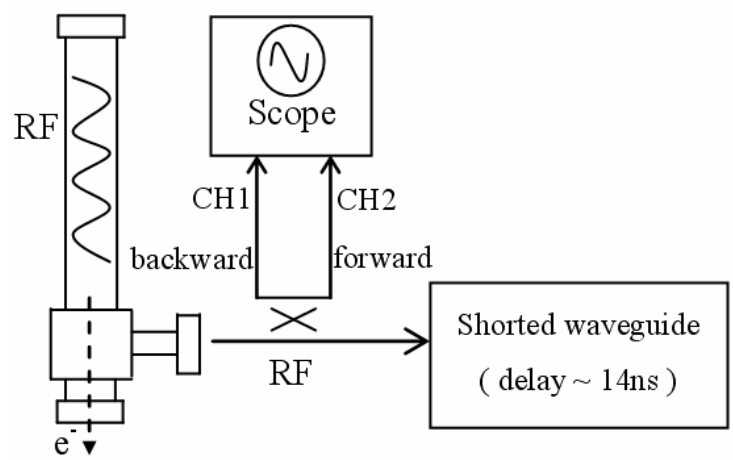

Figure 3: Beam experiment setup.

For the initial single beam test, the beam is carefully steered to travel through the power extractor, while the charge is varied by changing the intensity of the laser beam on the cathode. An integral current transformer (ICT) is placed right after the deceleration tube to monitor the charges pass the tube. So far the maximum charge detected by the ICT is $66 \mathrm{nC}$, which is $40 \%$ of that comes out from the gun. This is due to the fact that the structure is not placed in the optimal position for high charge transmission. The measured voltage signal is shown in Figure 4, where the total attenuation from bidirectional coupler, the cable and attenuators is $-90.9 \mathrm{~dB}$. The amplitude spectrum of the signal is shown in Figure 5, where it can be seen the frequency is right at $7.8 \mathrm{GHz}$.

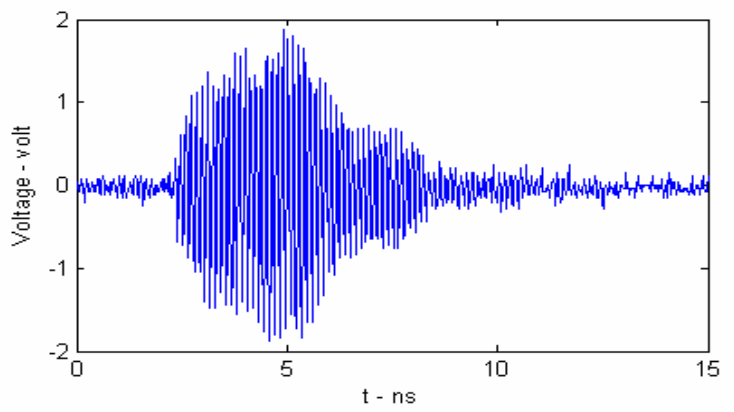

Figure 4: Voltage signal detected with the $15 \mathrm{GHz}$ oscilloscope $(q=66 \mathrm{nC})$. 


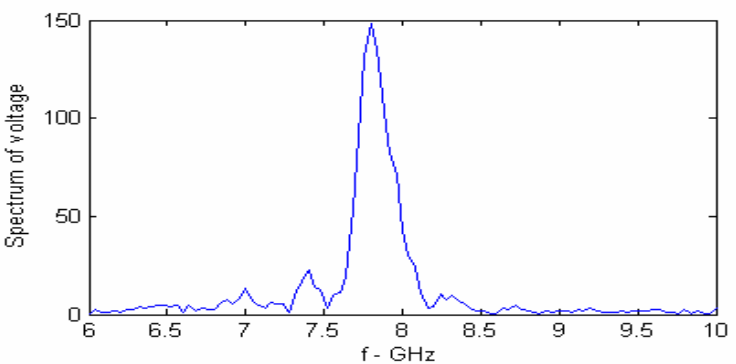

Figure 5: Spectrum of the voltage signal.

After correction on the attenuation, the generated power by the power extractor is experimentally obtained for different charge. Figure 6 shows both the measured and theoretical values, where a good agreement is found. It can be seen that when the charge is $66 \mathrm{nC}$, the generated power is $30 \mathrm{MW}$.

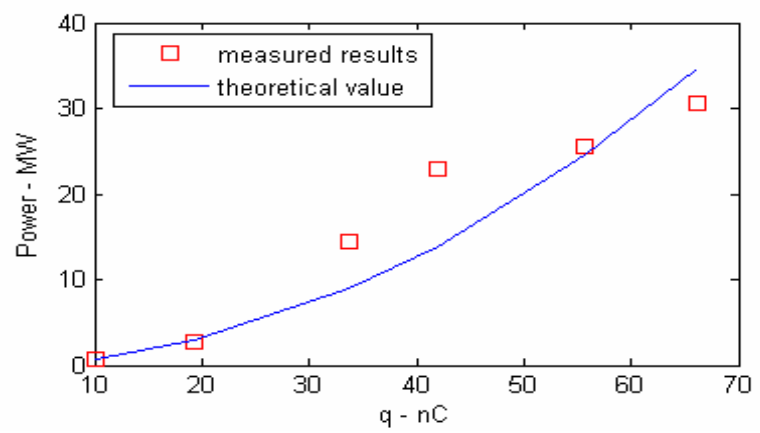

Figure 6: Generated power with different charge.

To test the bunch train excitation, we used a laser splitter set to generate a train consisting of four equal electron bunches. The time spacing between two adjacent bunches is $769 \mathrm{ps}$, therefore the bunch frequency is $1.3 \mathrm{GHz}$, the same as the linac frequency. Figure 7 clearly shows wakefield superposition by multiple bunches, where not only the amplitude is enhanced, but the pulse length is getting longer when more bunches are added in phase. For the signal shown in Figure 7(c), calculation shows the generated power is $211 \mathrm{KW}$, close to the theoretical prediction $216 \mathrm{KW}$. Figure 8 shows the spectrum of this signal.
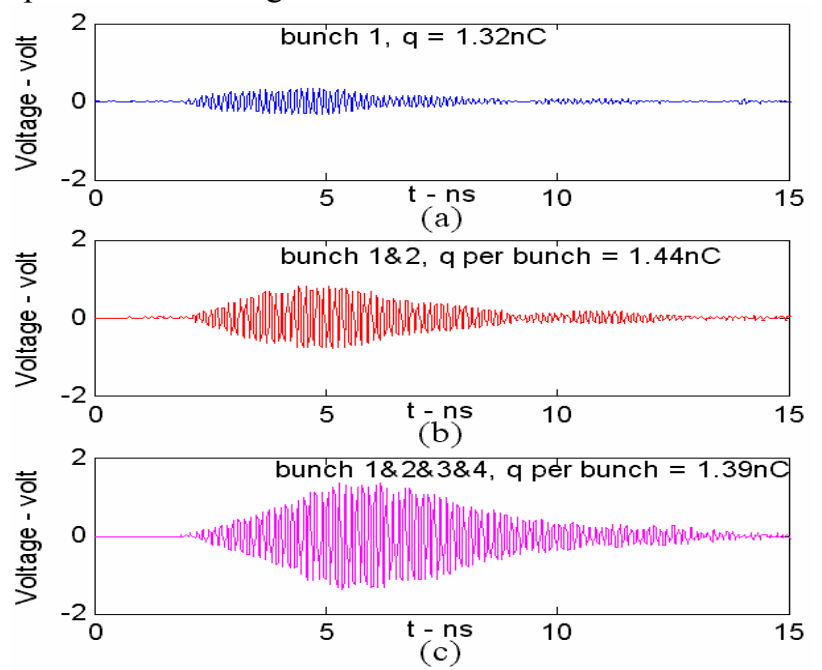

Figure 7: Measured excited voltage by bunches: (a) first bunch only; (b) first two bunches; (c) all four bunches.

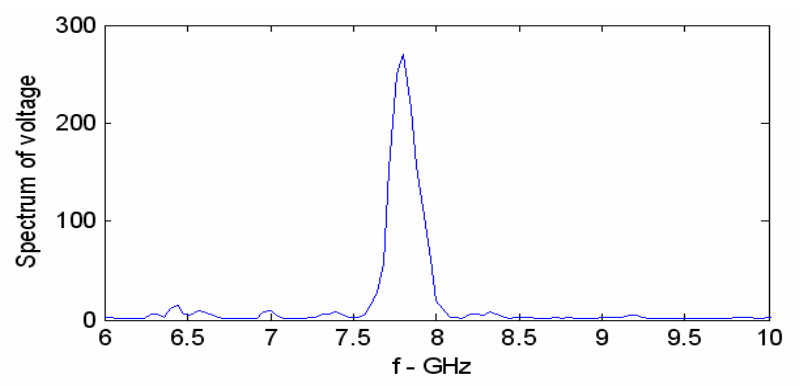

Figure 8: Spectrum of the voltage signal excited by the train consisting of four bunches shown in Figure 7(c).

\section{FUTURE PLAN}

We plan to move the power extractor further away from the gun, in order to send a shallower focused beam pass through the tube to improve charge transmission. Also a spectrometer will be used to monitor energy change of the decelerated beam, especially at high charge. Furthermore, we plan to generate a longer bunch train consisting of 16 bunches to get a longer RF pulse.

\section{SUMMARY}

The beam tests on the power extractor demonstrated the high power wakefield generation and extraction in a dielectric loaded acceleration structure. As a high power RF source in beam acceleration, this type of power extractors is promising due to its simplicity in design, low cost to fabricate, and ease to use.

\section{REFERENCES}

[1] G. Flesher and G. Cohn, AIEE Trans. 70, 887 (1951).

[2] J. Fang et al, Proc. PAC, 3627(1999).

[3] P. Zou et al, Review of Sci. Instr. 71, 2301(2000).

[4] R. Keinigs, M. Jones, and W. Gai, Part. Accel. 24, 223 (1989).

[5] T. B. Zhang, J. Hirshfield, T. Marshall, and N. Hafizi, Phys. Rev. E 56, 4647 (1997).

[6] W. Gai et al, PRL 61(1988).

[7] W. Gai, M. Conde et al, Proc. PAC, 1880(2001).

[8] D. Newsham et al, Proc. PAC, 1156(2003). 